\title{
THE EXISTENCE OF A MAXIMIZING VECTOR FOR THE NUMERICAL RANGE OF A COMPACT OPERATOR
}

\author{
URI FIXMAN, FRANK OKOH, AND G. K. R. RAO
}

(Communicated by Palle E. T. Jorgensen)

\begin{abstract}
Let $X$ be a complex Lebesgue space with a unique duality map $J$ from $X$ to $X^{*}$, the conjugate space of $X$. Let $A$ be a compact operator on $X$. This paper focuses on properties of $W(A)=\{J(x)(A(x)):\|x\|=1\}$ and $\Lambda(A)=\sup \{\operatorname{Re} \alpha: \alpha \in W(A)\}$. We adapt an example due to Halmos to show that for $X=l_{p}, 1<p<\infty$, there is a compact operator $A$ on $l_{p}$ with $W(A)$ the semi-open interval $[-1,0)$. So $\Lambda(A)$ is not attained as a maximum. A corollary of the main result in this paper is that if $X=l_{p}, 1<p<\infty$, and $\Lambda(A) \neq 0$, then $\Lambda(A)$ is attained as a maximum.
\end{abstract}

\section{INTRODUCTION}

A map $J: X \rightarrow X^{*}$ that satisfies $\|J(x)\|=\|x\|=\langle x, J(x)\rangle$ for all $x \in X$ is called a duality map for $X$. Here $\|x\|$ is the norm and $\langle$,$\rangle is the pairing defined by$ $\langle x, f\rangle=f(x)$ for $x \in X, f \in X^{*}$. We shall need a precise formula for the duality map for $L_{p}=L_{p}(\Omega, \Sigma, \mu), 1<p<\infty$, where $\Sigma$ is a $\sigma$-field of subsets of $\Omega$ and $\mu$ is an extended nonnegative countably additive measure on $\Sigma$. For such $p$ we identify $L_{p}^{*}$ with $L_{q}, q=p /(p-1)$. From Theorem IV, 8.1 of [6], we see that the linear functional on $L_{p}$ given by $x \in L_{q}$ is

$$
y \mapsto \int_{\Omega} y x d \mu, \quad y \in L_{p}
$$

We write $\langle y, x\rangle$ for $\int_{\Omega} y x d \mu$. For a nonzero complex number $\alpha, \operatorname{sgn} \alpha=\alpha /|\alpha|$ while $\operatorname{sgn} 0=0$. For $1<p<\infty$, put $K_{p}(\alpha)=|\alpha|^{p-1} \operatorname{sgn} \bar{\alpha}$. We extend this notation to functions $x: \Omega \rightarrow \mathbf{C}$ by $K_{p}(x)(\omega)=K_{p}(x(\omega))$ for all $\omega \in \Omega$. Since

$$
\left|K_{p}(x)\right|^{q}=|x|^{p}=x K_{p}(x)
$$

it follows that if $x \in L_{p}$, then $K_{p}(x) \in L_{q}$ and

$$
\left\|K_{p}(x)\right\|_{q}^{q}=\|x\|_{p}^{p}=\left\langle x, K_{p}(x)\right\rangle
$$

where $\|x\|_{p}$ is the norm of $L_{p}$. We define a map $J_{p}: L_{p} \rightarrow L_{q}$ by

$$
J_{p}(x)=\|x\|_{p}^{2-p} K_{p}(x) .
$$

From (3) we get

$$
\left\|J_{p}(x)\right\|_{q}^{2}=\|x\|_{p}^{2}=\left\langle x, J_{p}(x)\right\rangle .
$$

Received by the editors October 3, 1994; the contents of this paper were presented to the American Mathematical Society, October 25, 1974 .

1991 Mathematics Subject Classification. Primary 47A12. 
Therefore, $J_{p}$ is a duality map for $L_{p}$. It follows from Corollary 3.19 of [4] or [14] that $J_{p}$ is the only duality map for $L_{p}$. It is shown in [13] that neither $L_{1}$ nor $L_{\infty}$ has a unique duality map. Let $S(X)=\{x \in X:\|x\|=1\}$. For $x \in S\left(L_{p}\right)$ we have

$$
K_{p}(x)=J_{p}(x)
$$

and for $x \in L_{p}$,

$$
K_{q}\left(K_{p}(x)\right)=J_{q}\left(J_{p}(x)\right)=x .
$$

Let $A$ be a bounded linear operator on $X$. Put

$$
\psi_{A}(x)=\left\langle A x, K_{p}(x)\right\rangle .
$$

From (6) we get

$$
\Lambda(A)=\sup \left\{\operatorname{Re} \psi_{A}(x): x \in S\left(L_{p}\right)\right\} .
$$

In Theorem 11 of [11] it is shown that

$$
\Lambda(A)=\lim _{t \rightarrow 0^{+}} t^{-1}(\|I+t A\|-1)
$$

where $I$ is the identity operator on $X$. (See also Theorem 2 of [10].) When $\lambda=\Lambda(A)$ is attained as a maximum, a nonlinear eigenvalue problem for $\lambda$ is developed in [7]. Let $\rho(A)$ be the numerical radius of $A$. Then $\Lambda(A) \leq \rho(A)$. There are many papers on operators that attain their numerical radii. In some cases the set of such numerical radius attaining operators is norm-dense in the operator space of $X$; see [1], [2], and [12]. The reference for background is [3].

\section{1. $\Lambda(A)$ AS A MAXimum}

We begin with an example essentially due to Halmos in Problem 168, p. 111, of [9]. Let $\left(e_{j}\right)_{j=1}^{\infty}$ be the standard basis of $l_{p}, 1<p<\infty$. Let $A$ be the operator on $l_{p}$ whose matrix relative to this basis is diagonal with entries $-1 / n^{2}, n=1,2, \ldots$. One sees that $A$ is compact and $W(A)$ is the semi-open interval $[-1,0)$. Hence $\Lambda(A)=0$ and it is not attained as a maximum.

It is shown in [5] that if $A$ is a compact operator on a Hilbert space and $0 \in W(A)$, then $W(A)$ is closed. So in this case $\Lambda(A)$ is attained as a maximum. Even though our methods work for $l_{2}$ we shall slight this case because our results are not new for Hilbert spaces. On the other hand no extra effort is required to prove our main result for measure spaces, $(\Omega, \Sigma, \mu)$, in which every measurable subset of finite positive measure contains an atom. We recall that a measurable set $E$ is an atom in case $\mu(E)>0$ and for every measurable subset $F$ of $E$, either $\mu(F)=0$ or $\mu(E-F)=0$. The following lemma can be proved by imitating the proof of the Bolzano-Weierstrass theorem.

Lemma 1.1. Any measurable function on an atom is constant almost everywhere.

Lemma 1.2. Let $X=L_{p}(\Omega, \Sigma, \mu), 1<p<\infty$. Suppose that every measurable subset of finite positive measure contains an atom. Then $X$ has the following property: If $\left(x_{k}\right)_{k=1}^{\infty}$ is a sequence in $X$ which converges weakly to $x_{0}$, then $\left(K_{p}\left(x_{k}\right)\right)_{k=1}^{\infty}$ converges weakly to $K_{p}\left(x_{0}\right)$.

Proof. We use the FACT that $\left(x_{k}\right)_{k=1}^{\infty}$ converges weakly to $x_{0}$ if and only if

$$
\sup \left(\left\|x_{k}\right\|\right)_{k=1}^{\infty}<\infty
$$


and for every $E \in \Sigma$ with $0<\mu(E)<\infty$

$$
\lim _{k \rightarrow \infty} \int_{E} x_{k} d \mu=\int_{E} x_{0} d \mu
$$

See Ex. IV, 13.24 and Corollary III, 3.8 of [6].

Conditions (10) and (3) imply that

$$
\sup \left(\left\|K_{p}\left(x_{k}\right)\right\|_{q}\right)_{k=1}^{\infty}=M<\infty .
$$

Let $E \in \Sigma$ with $0<\mu(E)<\infty$. We first show that in verifying that the $K\left(x_{k}\right)$ satisfy the analogue of (11) we may replace $E$ by $E^{\prime}=\bigcup_{j=1}^{\infty} F_{j}$, where $E^{\prime} \subseteq E$ and $F_{j}$ is either an atom or the empty set, and $\mu\left(E^{\prime}\right)=\mu(E)$ : by hypothesis $E$ contains an atom $E_{1}$. If $\mu\left(E_{1}\right)=\mu(E)$, then $E^{\prime}=E_{1}$. Otherwise by Zorn's lemma there exists a maximal disjoint family $\left\{F_{j}: j \in \Gamma\right\}$ of atoms contained in $E$. Let $\Gamma_{n}=\left\{j \in \Gamma: \mu\left(F_{j}\right)>1 / n\right\}, n=1,2, \ldots$ As the cardinality of $\Gamma_{n}$ cannot exceed the finite number $n \mu(E)$ and $\Gamma=\bigcup_{n=1}^{\infty} \Gamma_{n}$, we conclude that $\Gamma$ is countable, taking it to be the set of positive integers. Let $G=E-\bigcup_{j=1}^{\infty} F_{j}$. If $\mu(G)>0$, then by our assumption, $G$ contains an atom contradicting the maximality of $\left\{F_{j}: j \in \Gamma\right\}$. Thus $\mu(G)=0$. Therefore in verifying the analogue of (11) for the $K\left(x_{k}\right)$ we may replace $E$ by $\bigcup_{j=1}^{\infty} F_{j}$, where we allow the possibility that some $F_{j}$ 's are empty. By Lemma $1.1, x_{k}$ is a constant $\alpha_{k j}$ on $F_{j}$ for each $j$. If $F_{j}$ is empty, put $\alpha_{k j}=0$. Thus we have for $k=0,1, \ldots$

$$
x_{k}=\sum_{j=1}^{\infty} \alpha_{k j} \chi_{F_{j}} .
$$

Since $x_{k}$ is integrable on $E, \alpha_{k j}<\infty$. Applying the FACT to each $F_{j}$, we obtain for $j=1,2, \ldots$

$$
\lim _{k \rightarrow \infty} \alpha_{k j}=\alpha_{0 j}
$$

Now we can prove that for $E$ as above,

$$
\lim _{k \rightarrow \infty} \int_{E} K\left(x_{k}\right) d \mu=\int_{E} K\left(x_{0}\right) d \mu
$$

$\left(K=K_{p}\right)$. Let $\varepsilon>0$ be given. Since $\mu(E)<\infty$, we can choose $l$ such that

$$
\mu\left(\bigcup_{j=l+1}^{\infty} F_{j}\right)<(\varepsilon /(4 M))^{p}
$$

Since $K$ is a continuous function on $\mathbf{C}$ into $\mathbf{C}$, it follows from (14) that we can choose $m \geq 1$ so that for $j=1,2, \ldots, l$ and all $k \geq m$

$$
\left|K\left(\alpha_{k j}\right)-K\left(\alpha_{0 j}\right)\right|<\varepsilon /\left(2 l \mu\left(F_{j}\right)\right) .
$$

Put $H=\bigcup_{j=l+1}^{\infty} F_{j}$. For all $k=1,2, \ldots$ we have

$$
\int_{E}\left(K\left(x_{k}\right)-K\left(x_{0}\right)\right) d \mu=\int_{H}\left(K\left(x_{k}\right)-K\left(x_{0}\right)\right) d \mu+\sum_{j=1}^{l}\left(K\left(\alpha_{k j}\right)-K\left(\alpha_{0 j}\right)\right) \mu\left(F_{j}\right) .
$$

Hence from (17),

$$
\left|\int_{E}\left(K\left(x_{k}\right)-K\left(x_{0}\right)\right) d \mu\right| \leq \varepsilon / 2+\int_{H}\left|K\left(x_{k}\right)\right| d \mu+\int_{H}\left|K\left(x_{0}\right)\right| d \mu .
$$


From Hölder's inequality we get that for $k=0,1,2, \ldots$

$$
\begin{aligned}
\int_{H}\left|K\left(x_{k}\right)\right| d \mu & \leq\left(\int_{H}\left|K\left(x_{k}\right)\right|^{q}\right)^{1 / q}\left(\int_{H} 1^{p}\right)^{1 / p} \\
& \leq\left\|K\left(x_{k}\right)\right\|_{q} \mu(H)^{1 / p} \leq M \cdot \varepsilon /(4 M)=\varepsilon / 4 .
\end{aligned}
$$

The last inequality is justified by (12) and (16). Therefore, we have from (18) that

$$
\left|\int_{E}\left(K\left(x_{k}\right)-K\left(x_{0}\right)\right) d \mu\right|<\varepsilon \text { for } k \geq m .
$$

This proves (15). By the FACT, (12) and (15) imply the required weak convergence of $K\left(x_{k}\right)$.

Before applying Lemma 1.2 let us show the necessity of the restriction on the measure space.

Proposition 1.3. Let $X=L_{p}(\Omega, \Sigma, \mu)$, where $1<p<\infty, p \neq 2$. Suppose that $\Omega$ contains a measurable set $E$ of finite positive measure such that $E$ contains no atom. Then there exists a sequence $\left(x_{k}\right)_{k=1}^{\infty}$ in $X$ converging weakly to $x_{0}$ such that $\left(K_{p}\left(x_{k}\right)\right)_{k=1}^{\infty}$ converges weakly to an element distinct from $K_{p}\left(x_{0}\right)$.

Proof. Since $E$ does not contain an atom, it follows from [8, p. 174] that $E$ can be partitioned into a disjoint union of two measurable subsets $E(1)$ and $E(2)$ with $\mu(E(1))=\mu(E(2))=\mu(E) / 2$. These sets satisfy the same hypothesis as $E$. Thus we find inductively a sequence of partitions of $E,\left\{E\left(j_{1}, j_{2}, \ldots, j_{k}\right): j_{m}=1\right.$ or $2\}, k=1,2, \ldots$, where the measure of a subset with $k$ indices is $2^{-k} \mu(E)$ and $E\left(j_{1}, \ldots, j_{k}\right)$ is the disjoint union of $E\left(j_{1}, \ldots, j_{k}, 1\right)$ and $E\left(j_{1}, \ldots, j_{k}, 2\right)$.

For each positive integer $k$, let $y_{k}$ be the function on $E$ which has the value $(-1)^{j_{1}+\cdots+j_{k}}$ on $E\left(j_{1}, \ldots, j_{k}\right)$. Denote by $\Sigma_{1}$ the $\sigma$-field generated in $E$ by all the sets $E\left(j_{1}, \ldots, j_{k}\right)$. Let $\mu_{1}$ be the restriction of $\mu$ to $\Sigma_{1}$. The sequence $\left(y_{k}\right)_{k=1}^{\infty}$ is a bounded sequence in $L_{p}\left(E, \Sigma_{1}, \mu_{1}\right)$ because $\left\|y_{k}\right\|_{p}=\mu(E)^{1 / p}$ for every $k$. If $F$ is any of the sets $E\left(j_{1}, \ldots, j_{m}\right)$ and $k>m$, then $\int_{F} y_{k} d \mu=0$. Thus,

$$
\lim _{k \rightarrow \infty} \int_{F} y_{k} d \mu=0 .
$$

The finite disjoint unions of the sets $E\left(j_{1}, \ldots, j_{m}\right)$ constitute a field $\Sigma_{0}$ in $E$, and (19) is valid for all $F \in \Sigma_{0}$. By [6, III, 8.3], the finite linear combinations of the characteristic functions of sets in $\Sigma_{0}$ are dense in $L_{p}\left(E, \Sigma_{1}, \mu_{1}\right)$. Hence it follows from $[6, I V, 13.24]$ that $\left(y_{k}\right)_{k=1}^{\infty}$ converges weakly to 0 in $L_{p}\left(E, \Sigma_{1}, \mu_{1}\right)$. Now the map which sends a function $f$ in $L_{p}\left(E, \Sigma_{1}, \mu_{1}\right)$ to its extension to $\Omega$ which vanishes on $\Omega-E$ is a linear isometry of $L_{p}\left(E, \Sigma_{1}, \mu_{1}\right)$ into $L_{p}(\Omega, \Sigma, \mu)$ (see, for example, [6, Lemma III, 8.1] and the following discussion). Denote the extension of $y_{k}$ also by $y_{k}$. So $\left(y_{k}\right)_{k=1}^{\infty}$ converges weakly to 0 in $L_{p}(\Omega, \Sigma, \mu)$. Replacing $p$ by $q$ we get the same conclusion for $L_{q}(\Omega, \Sigma, \mu)$.

Put $x_{k}=y_{k}+\chi_{E}, k=1,2, \ldots$. Then we have that $\left(x_{k}\right)_{k=1}^{\infty}$ converges weakly in $L_{p}(\Omega, \Sigma, \mu)$ to $\chi_{E}$. On the other hand, $K_{p}\left(x_{k}\right)=2^{p-2}\left(y_{k}+\chi_{E}\right)$ converges weakly to $2^{p-2} \chi_{E}$ which is distinct from $K_{p}\left(\chi_{E}\right)=\chi_{E}$.

Theorem 1.4. Let $X=L_{p}(\Omega, \Sigma, \mu)$ where $1<p<\infty$. Suppose that every measurable subset of $\Omega$ of finite positive measure contains an atom. Let $A$ be a compact operator on $X$ with $\Lambda(A) \neq 0$. Then there exists a vector $x$ in $S(X)$ such that $\operatorname{Re} \psi_{A}(x)=\Lambda(A)$. 
Proof. If $X$ is finite dimensional, then $S(X)$ is compact, by [6, p. 245]. Since $\operatorname{Re} \psi_{A}$ is continuous on $S(X)$, the conclusion holds in this case. If $X$ is infinite dimensional, then its identity operator is not compact and therefore $A$ cannot have a bounded inverse on $X$. So 0 is in the spectrum of $A$. According to [15, p. 217], the spectrum of a bounded linear operator, $A$, is contained in the closure of $W(A)$. Thus in the present case $\Lambda(A)>0$.

Given $\varepsilon$ such that $0<\varepsilon<\Lambda(A)$, there exists $y \in S(X)$ with $\Lambda(A)-\varepsilon \leq \operatorname{Re} \psi_{A}(y)$. Put $\operatorname{Re} \psi_{A}(y)=\alpha>0$ and $z=\alpha^{-1 / p} y$. As $K_{p}$ is positively homogeneous of degree $p-1, \psi_{A}$ is positively homogeneous of degree $p$. Thus $\operatorname{Re} \psi_{A}(z)=1$, and the set $E=\left\{u \in X: \operatorname{Re} \psi_{A}(u)=1\right\}$ is not empty. If we put

$$
\inf \{\|u\|: u \in E\}=\mu
$$

we have $\mu \leq\|z\|=\alpha^{-1 / p} \leq(\Lambda(A)-\varepsilon)^{-1 / p}$. Letting $\varepsilon$ tend to 0 , we get

$$
\mu \leq \Lambda(A)^{-1 / p} \text {. }
$$

We can obtain a sequence $\left(x_{k}\right)_{k=1}^{\infty}$ in $E$ such that $\lim _{k \rightarrow \infty}\left\|x_{k}\right\|=\mu$. Since $X$ is reflexive, the bounded sequence $\left(x_{k}\right)_{k=1}^{\infty}$ has a subsequence that converges weakly to some function $x_{0}$; see Lemma 28, p. 68 of [6]. We continue to denote this subsequence by $\left(x_{k}\right)_{k=1}^{\infty}$. For every $k=1,2, \ldots$ we have

$$
\begin{gathered}
\left|\operatorname{Re} \psi_{A}\left(x_{0}\right)-1\right|=\left|\operatorname{Re}\left\langle A x_{0}, K\left(x_{0}\right)\right\rangle-\operatorname{Re}\left\langle A x_{k}, K\left(x_{k}\right)\right\rangle\right|=R, \\
R \leq\left|\left\langle A x_{0}, K\left(x_{0}\right)\right\rangle-\left\langle A x_{k}, K\left(x_{k}\right)\right\rangle\right|=S, \\
S \leq\left|\left\langle A x_{0}, K\left(x_{0}\right)-K\left(x_{k}\right)\right\rangle\right|+\left|\left\langle A x_{0}-A x_{k}, K\left(x_{k}\right)\right\rangle\right| .
\end{gathered}
$$

By Lemma $1.2, K\left(x_{0}\right)-K\left(x_{k}\right)$ converges weakly to 0 as $k \rightarrow \infty$, which shows that the first summand on the right-hand side of (24) tends to 0. Since $A$ is compact, $A x_{k} \rightarrow A x_{0}$ strongly. Hence the second summand of (24), which by Hölder's inequality and (3) is dominated by $\left\|A x_{0}-A x_{k}\right\|_{p}\|x\|_{p}^{p-1}$, tends to 0 too. Therefore $x_{0} \in E$. So $x_{0} \neq 0$ and by $(20),\left\|x_{0}\right\| \geq \mu$. Since $\left(x_{k}\right)_{k=1}^{\infty}$ converges weakly to $x_{0}$, we have that $\left\|x_{0}\right\| \leq \lim _{k \rightarrow \infty} \inf \left\|x_{k}\right\|$; see Lemma 27, p. 68 of [6]. We get from $\lim _{k \rightarrow \infty}\left\|x_{k}\right\|=\mu$ that $\left\|x_{0}\right\| \leq \mu$. Hence, $\left\|x_{0}\right\|=\mu$ and $\mu>0$. Put $x=\mu^{-1}\left(x_{0}\right)$ so that $x \in S(X)$. We have that $\operatorname{Re} \psi_{A}(x)=\mu^{-p} \operatorname{Re} \psi\left(x_{0}\right)=\mu^{-p} \geq \Lambda(A)$, where the last inequality comes from (21). From (9) we now conclude that $\operatorname{Re} \psi_{A}(x)=$ $\Lambda(A)$.

Remark. (a) We do not know whether the restriction on the measure, which is necessary in Lemma 1.2, is also necessary in Theorem 1.4.

(b) There exist noncompact operators $B$ on $l_{p}$ with $\Lambda(B)>0$ for which $\Lambda(B)$ is attained as the maximum of $\operatorname{Re} \psi_{B}$ in $S\left(l_{p}\right)$, and also such that it is not attained. For the former it suffices to take $B=A+\alpha I$, where $\alpha>0$ and $A$ satisfies the assumptions of Theorem 1.4. Examples of such $A$ abound, see for instance [5]. For the latter take $B=A+\alpha I$, where $\alpha>0$ and $A$ is the operator mentioned in the beginning of the section.

\section{REFERENCES}

1. M. D. Acosta and R. Payá, Numerical radius attaining operators and the Radon-Nikodym property, Bull. London Math. Soc. 25 (1993), 67-73. MR 93j:47005

2. I. Berg and B. Sims, Denseness of numerical radius attaining operators, J. Austral. Math. Soc. Ser. A 36 (1984), 130-133. MR 84j:47004

3. F. F. Bonsall and J. Duncan, Numerical ranges of operators on normed spaces, I, II Cambridge Univ. Press, London and New York, 1971, 1973. MR 44:5779 
4. D. F. Cudia, The geometry of Banach spaces. Smoothness, Trans. Amer. Math. Soc. 110 (1964), 284-314. MR 29:446

5. G. De Barra, J. R. Giles, and B. Sims, On the numerical range of compact operators on Hilbert spaces, J. London Math. Soc. (2) 5 (1972), 702-706. MR 47:4044

6. N. Dunford and J. T. Schwartz, Linear operators, Part I, General theory, Interscience, New York, 1958. MR 22:8302

7. U. Fixman, F. Okoh, and G. K. R. Rao, An eigenvalue problem for the numerical range of a bounded linear transformation, preprint.

8. P. R. Halmos, Measure theory, Van Nostrand, New York, 1950. MR 11:504d

9. __ Hilbert space problem book, Van Nostrand, New York, 1967. MR 34:8178

10. L. A. Harris, The numerical range of holomorphic functions in Banach spaces, Amer. J. Math. 93 (1971), 1005-1019. MR 46:663

11. G. Lumer, Semi-inner product spaces, Trans. Amer. Math. Soc. 100 (1961), 29-43. MR 24:a2860

12. R. Payá, A counterexample on numerical radius attaining operators, Israel J. Math. 79 (1992), 83-101. MR 93j:47004

13. G. K. R. Rao, Numerical ranges of linear operators in $L_{p}$-spaces, Doctoral thesis, Queen's University, Kingston, Canada, 1974.

14. V. L. Smŭlian, Sur la dérivabilité de la norme dans l'espace de Banach, Dokl. Akad. Nauk SSSR 27 (1940), 643-648. MR 2:102f

15. J. P. Williams, Spectra of products and numerical ranges, J. Math. Anal. Appl. 17 (1967), 214-220. MR 34:3341

(U. Fixman and G. K. R. Rao) Department of Mathematics and Statistics, Queen's University, Kingston, Ontario, Canada K7L 3N6

(F. Okoh) Department of Mathematics, Wayne State University, Detroit, Michigan 48202

E-mail address: okoh@math.wayne.edu 